\title{
Clodronate inhibits angiogenesis in vitro and in vivo
}

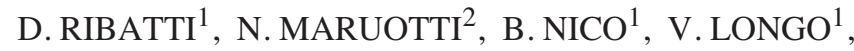

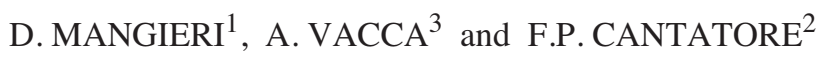 \\ ${ }^{1}$ Department of Human Anatomy and Histology, University of Bari Medical School, Bari; \\ ${ }^{2}$ Department of Rheumatology, University of Foggia Medical School, Foggia; ${ }^{3}$ Department of \\ Biomedical Sciences and Clinical Oncology, University of Bari Medical School, Bari, Italy
}

Received October 1, 2007; Accepted December 4, 2007

\begin{abstract}
The effects of amino-bisphosphonate clodronate on endothelial cell functions involved in angiogenesis, namely proliferation and morphogenesis on matrigel were tested in vitro, whereas its effects on angiogenesis were studied in vivo. This was performed by using the chick embryo chorioallantoic membrane (CAM) assay. In vitro, clodronate inhibited the endothelial cell proliferation in a dose-dependent fashion, peaking at $30 \mu \mathrm{M}$. At the same concentration, clodronate inhibited the fibroblast growth factor-2 (FGF-2)induced capillary-like tube formation in the morphogenesis assay on matrigel. In vivo, when tested with the CAM assay, clodronate again displayed the capability to inhibit FGF-2induced angiogenesis. Overall, these results suggest that antiangiogenesis by clodronate can be used to treat a wide spectrum of angiogenesis-dependent diseases, including certain chronic inflammatory diseases and cancer.
\end{abstract}

\section{Introduction}

Bisphosphonates (BP) are analogues of PPi and differ from one another based on their substituted side chain (1). They can be segregated into two distinct pharmacological classes [i.e., nitrogen containing (amino) and non-nitrogenic-containing bisphosphonates] based on their molecular mechanism of action (2).

Some evidence suggests that part of the antitumor activity of bisphosphonates may be attributed to an antiangiogenic effect. Wood et al (3) reported that endothelial cell proliferation and migration in vitro induced by the fibroblast growth factor-2 (FGF-2), the vascular endothelial growth factor (VEGF) and angiogenesis in vivo in the chick embryo chorioallantoic membrane (CAM) assay are inhibited by

Correspondence to: Professor Domenico Ribatti, Department of Human Anatomy and Histology, Policlinico, Piazza Giulio Cesare 11, I-70124 Bari, Italy

E-mail: ribatti@anatomia.uniba.it

Key words: antiangiogenesis, bisphosphonate, chorioallantoic membrane, clodronate, endothelial cells zoledronic acid. The same investigators demonstrated that in a subcutaneous growth factor implant model in mice, zoledronate treatment strongly inhibited the angiogenic response induced by FGF-2 and VEGF (4).

Fournier et al (5) analyzed the role of bisphosphonates on the inhibition of endothelial cell functions both in vitro and in vivo. Four bisphosphonates were tested in vitro: clodronate, ibandronate, risedronate and zoledronic acid. In vivo studies were performed by quantification of the vascularization in bone biopsy specimens from patients with Paget's disease before and after clodronate treatment and by analysis of testosterone-induced revascularization of the prostate gland in castrated rats. Santini et al (6) showed a significant decrease of circulating levels of VEGF in cancer patients with bone metastases receiving a single dose of pamidronate. Bezzi et al (7) showed that zoledronate inhibited endothelial cell adhesion and migration and enhanced tumor necrosis factor-mediated endothelial cell death. We previously demonstrated that neridronate inhibited angiogenesis in vitro and in vivo (8).

Herein, we examined the effects of clodronate in the early phases of the angiogenesis process, i.e. endothelial cell proliferation and in the late differentiative phases of neovascularization, i.e. formation of capillary-like structures by endothelial cells seeded on matrigel. In vitro observations were compared with the effects exerted in vivo by clodronate on physiological angiogenesis in the CAM assay, a useful model for such an investigation (9). The results demonstrate that clodronate inhibits angiogenesis in vitro and affects blood vessel formation in vivo.

\section{Materials and methods}

Cells and preparation of conditioned media (CM). Human umbilical vein endothelial cells (HUVECs) were prepared as previously described (10). The cells were grown in Petri dishes coated with $1 \%$ gelatin (Sigma Chemical Co., St. Louis, MO, USA) in a complete M199 medium (Seromed) supplemented with $20 \%$ heat-inactivated fetal calf serum (FCS) (Seromed), $0.02 \%$ bovine brain extract and $0.01 \%$ porcine heparin (both Sigma).

Proliferation assay. HUVECs $\left(4 \times 10^{3}\right.$ cells/well) were plated in a 96-well plate (Falcon 3072, Becton Dickinson, Mountain 
View, CA, USA) in a complete medium. After $24 \mathrm{~h}$ of incubation, the medium was replaced with a fresh one, containing clodronate (Abiogen, Pisa, Italy) at different doses $(1,3,10,30$ and $50 \mu \mathrm{M})$ alone or with recombinant human FGF-2 (R and D Systems, Abingdon, UK) (50 ng/ml) for an additional $24 \mathrm{~h}$. Cell numbers were trypsinized and estimated by the crystal violet colorimetric assay of Kueng et al (11) read at $595 \mathrm{~nm}$ in the Microplate reader modl 3550 (Bio-Rad Laboratories, Richmond, CA, USA). The cell number was expressed as a mean \pm 1 SD of six separate experiments.

Morphogenesis assay on matrigel. The assay assesses the ability of endothelial cells to produce 'spontaneous angiogenesis in vitro', i.e. a three-dimensional vascular tube and cord-like structure connecting 'cellular nodes' and resembling an organized capillary mesh. HUVECs cells $\left(1.2 \times 10^{5} /\right.$ well $)$ were plated in duplicate in 24 -well plates $\left(2 \times 10^{5}\right.$ cells/well) precoated with matrigel (300 $\mu 1 /$ well; Becton Dickinson) in $1 \mathrm{ml} / \mathrm{well}$ in a basal medium, containing or not FGF-2 $(50 \mathrm{ng} / \mathrm{ml})$ or clodronate at the same doses tested in the proliferation assay. After $18 \mathrm{~h}$ of incubation at $37^{\circ} \mathrm{C}$, cell growth and tridimensional organization were observed through a reverted phase-contrast light microscope.

Chorioallantoic membrane (CAM)-gelatin sponge assay and measurement of angiogenesis. Fertilized white leghorn chicken eggs (20 per group) were incubated under conditions of constant humidity at $37^{\circ} \mathrm{C}$. On day 3 of incubation, a square window was opened in the shell after removal of 2-3 $\mathrm{ml}$ of albumen to allow detachment of the developing CAM. The window was sealed with a glass and the eggs were returned to the incubator. CAMs were treated on day 8 with $1 \mathrm{~mm}^{3}$ gelatin sponges (Gelfoam; Upjohn Co, Kalamazoo, MI, USA) (9) containing vehicle alone (PBS) and were used as negative controls, or with $500 \mathrm{ng} / \mathrm{embryo}$ of FGF-2 dissolved in $2 \mu \mathrm{l}$ of PBS, as positive controls. In some experiments, FGF-2 was added to $50 \mu \mathrm{M}$ clodronate dissolved in $2 \mu 1$ of PBS. All procedures were performed under sterile conditions. CAMs were examined daily until day 12 and photographed in ovo with a Zeiss SR stereomicroscope equipped with the MC63 camera system (Zeiss, Oberkochen, Germany). Blood vessels entering the sponges within the focal plane of the CAM were counted by two observers in a double blinded fashion at a magnification of $\mathrm{x} 50$.

\section{Results}

Effects of clodronate on the angiogenic phenotype of endothelial cells. The first series of experiments focused on the effects of clodronate on the proliferation of HUVECs. As shown in Fig. 1, clodronate reduced endothelial cell growth in a dose-dependent fashion peaking at $30 \mu \mathrm{M}$, whereas $50 \mu \mathrm{M}$ gave a plateau.

In the second series, the effect of clodronate on capillary morphogenesis was investigated. After seeding on matrigel HUVECs in the presence of FGF-2 (50 ng/ml), HUVECs formed a rich meshwork of branching anastomosing capillary-like tubules with multicentric junctions (Fig. 2A). When FGF-2 was administered in the presence of clodronate $(30 \mu \mathrm{M})$, the capillary-like tubes were interrupted and most

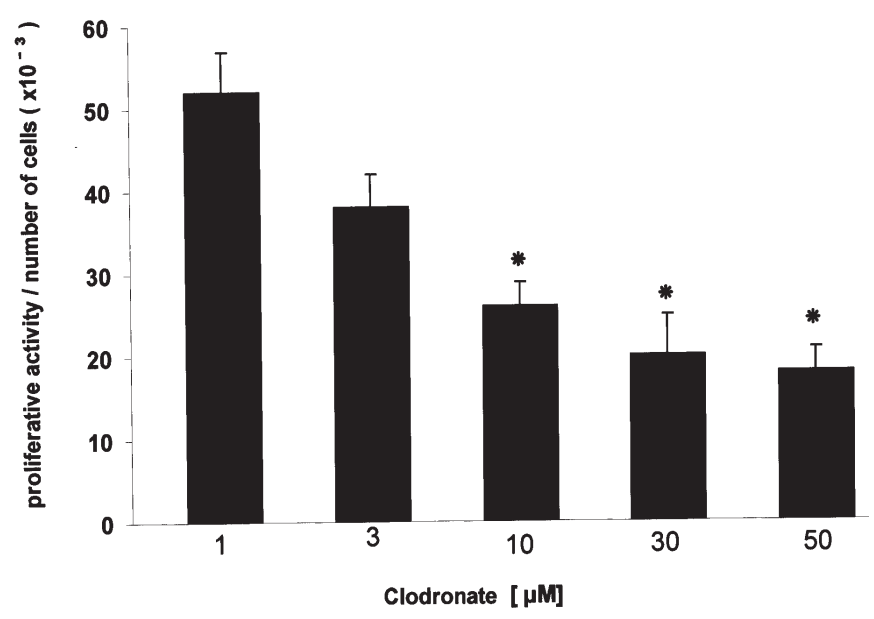

Figure 1. Effects of clodronate tested at different concentrations on the proliferative activity of HUVECs. Bars are means \pm SD $(n=6)$.

cells were spherical, either isolated or aggregated in small clumps with only a few cells elongated (Fig. 2B).

Inhibition of CAM vascularization by sponges treated with clodronate. Gross inspection on day 12 of incubation showed that the gelatin sponges treated with FGF-2 (positive control) were surrounded by allantoic vessels that developed radially in a 'spoked wheel' pattern towards them (mean number of vessels $=27 \pm 4)($ Fig. 3A). When the sponges were loaded with PBS (negative control), physiological angiogenesis was observed in the form of some allantoic vessels partly arranged

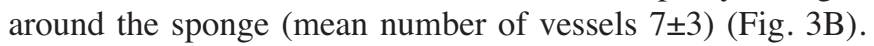
On day 12 of incubation, when sponges treated with FGF-2 were supplemented with clodronate administered at $50 \mu \mathrm{M} /$ embryo, a significant reduction of FGF-2-induced angiogenic response was appreciable macroscopically (mean number of vessels 14 \pm 3 ) and microscopically (Fig. 3C).

\section{Discussion}

The existence of specific angiogenesis inhibitors was first postulated by J. Folkman in 1971 (12). The term 'antiangiogenesis' was introduced in order to describe treatment designed to prevent the induction of new blood vessels and perhaps to reduce the number of those already present.

Three strategies block tumor growth in experimental models through the regression of angiogenesis: vascular targeting, gene therapy and the direct inhibition of proliferating and migrating endothelial cells. Alternatively, indirect antiangiogenic drugs prevent expression or block the activity of tumor proangiogenic factors by interfering with their endothelial receptors.

Inhibitors of angiogenesis block any of the several steps in the angiogenic cascade. This includes proliferation and attachment of endothelial cells to the extracellular matrix proteins, migration and invasion through the matrix, which is required for the capillary sprouting and morphogenesis in a thin tube meshwork and differentiation and stabilization (13).

Several in vitro and in vivo studies indicate that bisphosphonates have antiangiogenic properties that could 

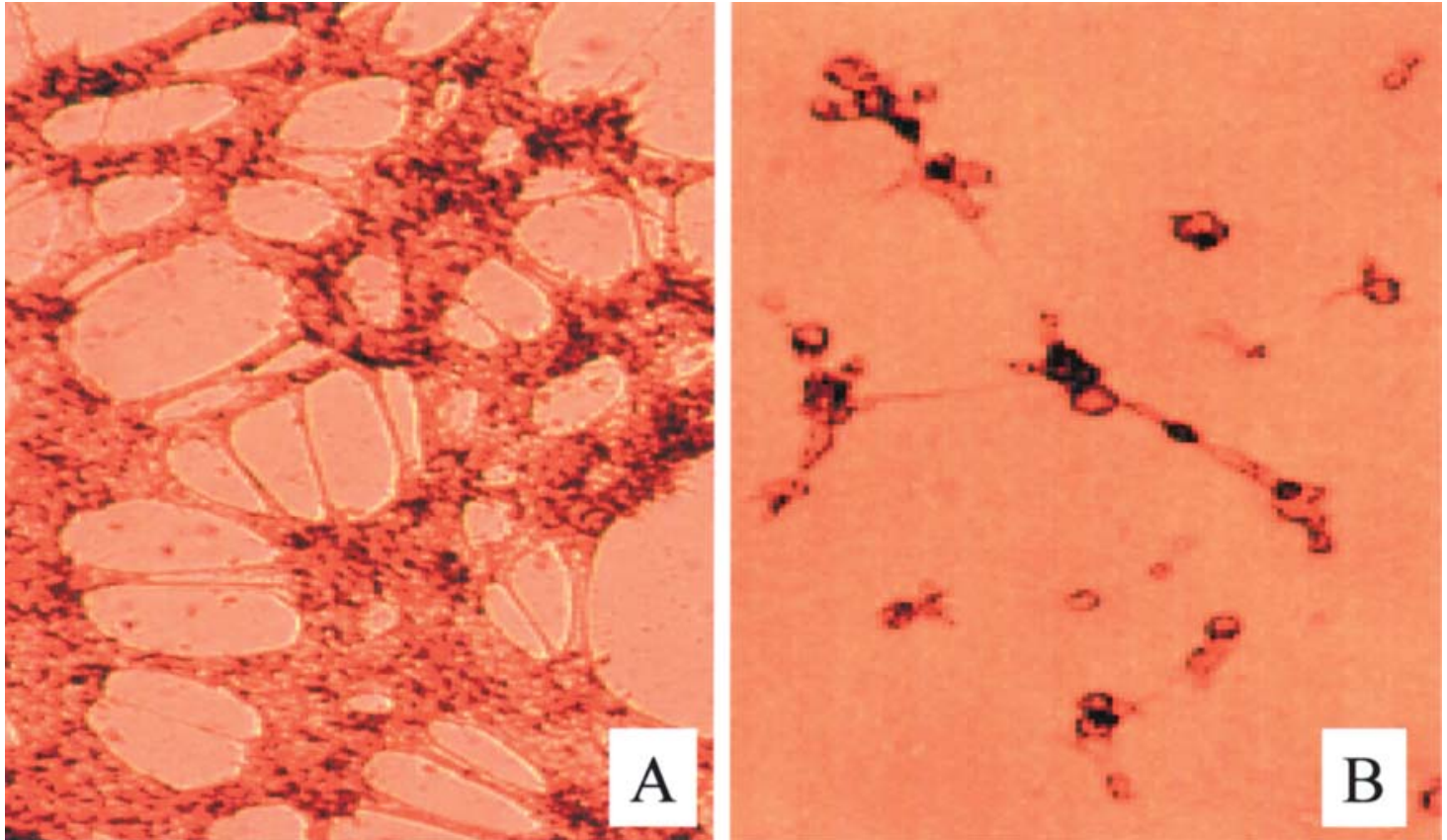

Figure 2. Phase contrast micrographs illustrating the arrangements of HUVECs into a rich meshwork of capillary-like tubular structures when cultured on Matrigel for $18 \mathrm{~h}$ in the presence of FGF-2 (A). The disrupting effect of clodronate (B). Magnification, x50.
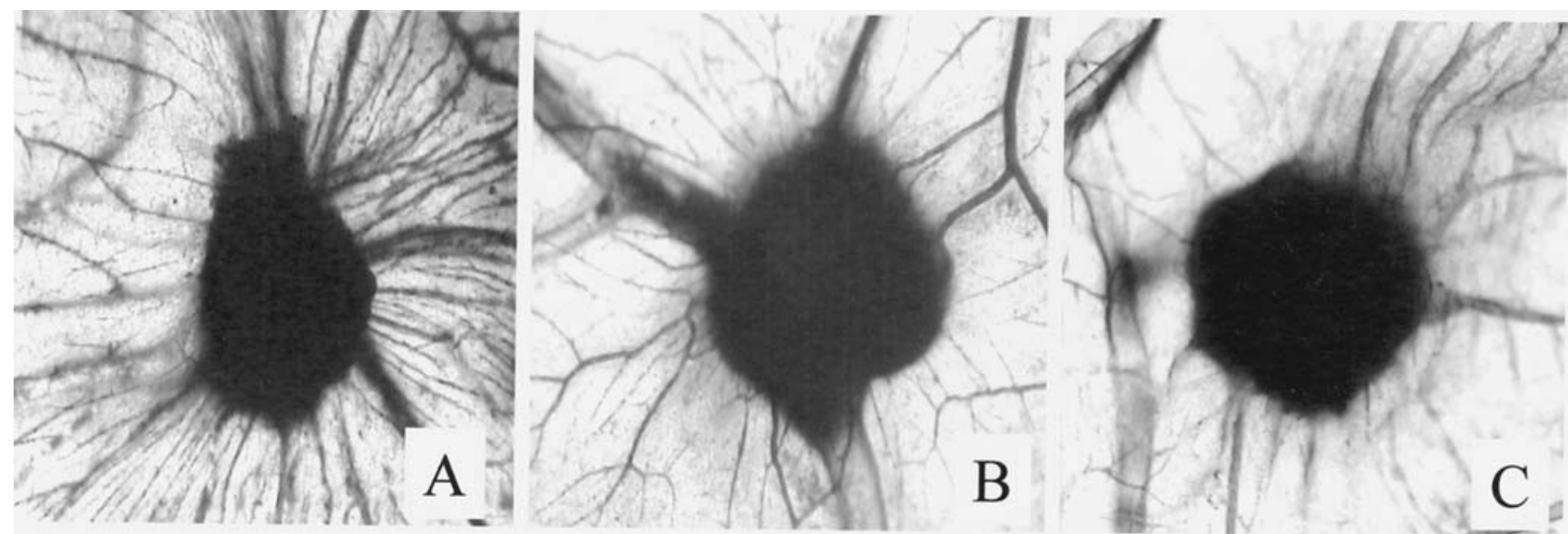

Figure 3. In vivo CAM assay. (A) Macroscopic observations on day 12 of incubation shows that the sponges adsorbed with FGF-2 are surrounded by allantoic vessels developing radially towards the implant in a 'spoked-wheel' pattern. (B) No vascular reaction is detectable around the vehicle-treated sponge. (C) When FGF-2 is administered in combination with clodronate, a significant reduction of the angiogenic response is appreciable. Magnification, $\mathrm{x} 50$.

contribute to their efficacy in the treatment of malignant bone diseases and extend their potential clinical use to other cancers and diseases with an angiogenic component, such as inflammatory diseases.

We demonstrated that clodronate is an inhibitor of angiogenesis in vitro, reducing the endothelial growth and morphogenesis on matrigel. These in vitro effects were matched by the inhibition of angiogenesis in vivo in the CAM, where clodronate inhibited FGF-2-induced angiogenesis.

BP are potent inhibitors of bone resorption and are widely used drugs for treatment of osteoporosis and bone metastasis (14). In vitro anti-tumor properties of BP include inhibition of tumor proliferation and invasion and induction of apoptosis of various cancer cell lines, especially when combined with other standard antineoplastic therapies (15). In vivo, this antitumor effect appears to be limited to tumor cells in bone metastases, at least in the majority of experiments performed to date (15).

$\mathrm{BP}$ has been reported to accumulate in human vessels (16). Furthermore, nitrogen-containing BP such as ibandronate and zoledronic acid have been shown to inhibit angiogenesis in vitro and in vivo in several assays $(3,16)$ or showed that other BP antagonize angiogenesis and tumor growth in traditional subcutaneous xenotransplant tumor models $(17,18)$.

Yamagishi et al (19) demonstrated that minodronate, a newly developed nitrogen-containing BP, can inhibit melanoma growth and improve survival in nude mice by suppressing the tumor-associated angiogenesis and macrophage infiltration in vivo. Moreover, they also found that minodronate blocked the angiogenesis signaling in microvascular endothelial cells. Zeisberger et al (20) showed 
that clodronate-liposome-mediated tumor associated macrophage depletion inhibited tumor growth, presumably through the blocking of tumor angiogenesis.

In recent years, studies based on human clinical models have been performed in order to confirm the antiangiogenic reports obtained by in vitro and in vivo preclinical studies (21).

Taken together, our results indicate that clodronate exerts its antiangiogenic activity through a direct effect on endothelial cell proliferative activity and an inhibitory effect on the responsivity of the endothelial cells to the proliferative stimuli mediated by an angiogenic cytokine, such as FGF-2. This suggests that clodronate, alone or in combination with other therapeutic strategies, may provide new opportunities to treat a wide spectrum of angiogenesis-dependent diseases, including certain chronic inflammatory diseases and cancer.

\section{Acknowledgements}

This study was supported by MIUR (PRIN 2005), Rome and Abiogen, Pisa, Italy.

\section{References}

1. Shinada H, Adamek G, Felix R, Fleisch H, Schenk R and Hagon H: Structure-activity relationships of various bisphophonates. Calcif Tissue Int 35: 87-99, 1983.

2. Benford HL, Fith JC, Auriola S, Monkkonen J and Bogers MJ: Farnesol and geranylgeraniol prevent activation of caspase by aminobisphosphonates: biochemical evidence for two distinct pharmacological classes of bisphosphonate drugs. Mol Pharmacol 56: 131-140, 1999.

3. Wood J, Bonjean K, Ruetz S, Bellahcene A, Devy L, Foidart JM, Castronovo V and Green JR: Novel antiangiogenic effects of the bisphosphonate compound zoledronic acid. J Pharmacol Exp Ther 302: 1055-1061, 2002.

4. Wood J, Schnell C and Green JR: Zoledronic acid, a potent inhibitor of bone resorption inhibits proliferation and induces apotosis in human endothelial cells in vitro and is anti-angiogenic in a murine growth factor implant model. Proc Am Soc Clin Oncol 9: 664 Abs. 2620, 2000.

5. Fournier P, Boissier S, Filleur S, Guglielmi J, Cabon F, Colombel $\mathrm{M}$ and Clezardin P: Bisphosphonates inhibit angiogenesis in vitro and testosterone-stimulated vascular regrowth in the ventral prostrate in castrated rats. Cancer Res 62: 6538-6544, 2002.

6. Santini D, Vincenzi B, Avvisati G, Dicuonzo G, Battistoni F, Gavasci M, Salerno A, Denaro V and Tonini G: Pamidronate induces modifications of circulating angiogenic factors in cancer patients. Clin Cancer Res 8: 1080-1094, 2002.
7. Bezzi M, Hasmim M, Bieler G, Dormond O and Ruegg C: Zoledronate sensitizes endothelial cells to tumor necrosis factorinduced programmed cell death: evidence for the suppression of sustained activation of focal adhesion kinase and protein kinase B/Akt. J Biol Chem 44: 43603-43614, 2003.

8. Ribatti D, Nico B, Mangieri D, Maruotti N, Longo V, Vacca A and Cantatore FP: Neridronate inhibits angiogenesis in vitro and in vivo. Clin Rheumatol 26: 1094-1098, 2007.

9. Ribatti D, Nico B, Vacca A and Presta M: The gelatin spongechorioallantoic membrane assay. Nat Protocols 1: 85-91, 2006.

10. Vacca A, Iurlaro M, Ribatti D, Minischetti M, Nico B, Pellegrino A and Dammacco F: Angiogenesis is produced by non-toxic doses of vinblastine. Blood 94: 4143-4155, 1999.

11. Kueng K, Silfer E and Eppenberger V: Quantitation of cells cultured on 96-well plates. Ann Biochem 182: 16-19, 1989.

12. Folkman J: Tumor angiogenesis: therapeutic implications. N Engl J Med 285: 1182-1186, 1971.

13. Folkman J: Angiogenesis in cancer, vascular, rheumatoid and other disease. Nat Med 1: 27-31, 1995.

14. Brown DL and Robbins R: Developments in the therapeutic applications of bisphosphonates. J Clin Pharmacol 39: 651-660, 1999.

15. Green JR: Antitumor effects of bisphosphonates. Cancer 97 (Suppl 3): 840-847, 2003

16. Ylitalo R, Kalliovalkama J, Wu X, Kankaanranta H, Salenius JP, Sisto T, Lahteenmaki T, Ylitalo P and Porsti I: Accumulation of bisphosphonates in human artery and their effects on human and rat arterial function in vitro. Pharmacol Toxicol 83: 125-131, 1998.

17. Hamma-Kourbali Y, Di Benedetto M, Ledoux D, Oudar O, Leurox Y, Lecouvey M and Kraemer M: A novel non-containingnitrogen bisphosphonate inhibits both in vitro and in vivo angiogenesis. Biochem Biophys Res Commun 310: 816-823, 2003.

18. Sebbah-Louriki M, Colombo BM, el Manouni D, Martin A, Salzmann JL, Leroux Y, Perret GY and Crepin M: A new phenylacetate-bisphoshonate inhibits breast cancer cell growth by proapoptotic and antiangiogenic effects. Anticancer Res 22: 3925-3931, 2002.

19. Yamagishi S, Abe R, Inagaki Y, Nakamura K, Sugawara H, Inokuma D, Nakamura H, Shimizu T, Takeuchi M, Yoshimura A, Bucala R, Shimizu H and Imaizumi T: Minodronate, a newly developed nitrogen-containing bisphosphonate, suppresses melanoma growth and improves survival in nude mice by blocking vascular endothelial growth factor signaling. Am J Pathol 165: 1865-1874, 2004.

20. Zeisberger SM, Odermatt B, Marty C, Zehnder AHM, Ballmer-Hofer K and Schwendener RA: Clodronate-liposomemediated depletion of tumour-associated macrophages: a new and highly effective antiangiogenic therapy approach. Br J Cancer 95: 272-281, 2006.

21. Vincenzi B, Santini D, Dicuonzo G, Battistoni F, Gavasci M, La Cesa A, Grilli C, Virzi V, Gasparro S, Rocci L and Tonini G: Zoledronic acid-related angiogenesis modifications and survival in advanced breast cancer patients. J Interferon Cytokine Res 25: 144-151, 2005. 